\title{
Ambient Intelligence and Pervasive Systems for the Monitoring of Citizens at Cardiac Risk: New Solutions from The EPI-MEDICS Project
}

\author{
F Gouaux ${ }^{1}$, L Simon-Chautemps ${ }^{1}$, J Fayn ${ }^{1}$, S Adami ${ }^{2}$, M Arzi ${ }^{1}$, D Assanelli ${ }^{3}$, MC Forlini ${ }^{1}$, \\ C Malossi, A Martinez ${ }^{4}$ J Placide $^{1}$, GL Ziliani ${ }^{2}$, P Rubel $^{1}$ \\ ${ }^{1}$ INSERM, Lyon, France, ${ }^{2}$ Elettronica Trentina, Italy, ${ }^{3}$ University of Brescia, Italy, \\ ${ }^{4}$ STMicroelectronics, Italy
}

\begin{abstract}
In western countries, heart disease is the main cause of premature death. Most of cardiac deaths occur out of hospital. Symptoms are often interpreted incorrectly. Victims do not survive long enough to benefit from inhospital treatments. To reduce the time before treatment, the only useful diagnostic tool to assess the presence of a cardiac event is the electrocardiogram (ECG). Event and transtelephonic ECG recorders are used to improve decision-making but require setting up new infrastructures. The pervasive solution proposed by the European EPI-MEDICS project is an intelligent Personal ECG Monitor for the early detection of cardiac events. It includes decision-making techniques, generates different alarm levels and forwards alarm messages to the relevant care providers by means of new generation wireless communication. It is cost saving, involving care provider only if necessary without specific infrastructure. Healthcare becomes personalized, wearable, ubiquitous.
\end{abstract}

\section{Introduction}

The past years saw the progress of Medical Informatics. But after decades of development of information systems dedicated to health professionals, there is an increasing demand for personalized and nonhospital based care [1].

Besides, new classes of computing and communication devices like smart cards, handheld computers (PDAs) and smart phones have emerged. These recent devices together with the advances of wireless technologies will allow the citizens to access to their data anywhere and anytime. This concept is called Pervasive Computing [2]. This includes entertainment systems such as MP3 players, household appliances, point-of-sales terminals, medical monitoring devices...

Another recent concept is Ambient Intelligence. Defined by the EC Information Society Technologies Advisory Group in 1999, this idiom describes a potential future in which we will be surrounded by intelligent objects and in which the environment will recognize the presence of persons and will respond to it in an undetectable manner [3]. Ambient Intelligence is based on three recent key technologies:

- Ubiquitous Computing that describes the fact that microprocessors are embedded into everyday objects and so, that many computers are made available throughout the physical environment while being invisible to the user [4],

- Ubiquitous Communication that enables these objects to communicate by means of wireless technologies with other devices, hosts and users,

- Intelligent User Interfaces that enables the citizens to interact with such an intelligent environment not only in a natural way but also in a personalized way.

In a near future, citizens and patients will use such new technologies to access information and above all, to control their own health care acting as consumers responsible of their own health. They will be able to perform relevant tests without involving skilled personnel from the beginning, but only when a specialized action is required. Healthcare is moving from traditional institution-centered care towards a patient, citizencentered one via the use of ubiquitous and pervasive systems. Professional care will be increasingly viewed as the support to systems that emphasize self care [5].

This general trend is especially perceptible in the field of cardiology. It is known that heart disease is the main cause of early disability and premature death in western countries. Persons with acute myocardial infarction (AMI) who survive long enough to enter hospital undoubtedly benefit from the improvement of the quality of care introduced into routine practice within the last decade. But the majority of deaths from coronary disease occur in the pre-hospital phase and most victims do not survive long enough to receive medical help [6].

During previous European Framework Programmes, several solutions have been designed to improve cardiac patient health by providing seamless patient information availability and sharing throughout Europe. Attempts have been made to improve the management of cardiac care: campaigns to teach symptoms of heart attacks, use of the agreed common European emergency number 
(112), strategic positioning of ambulances, pre-hospital triage and arrangements for cardiac care based on advanced information from the ambulance or the patient [7]. Nevertheless, only small trends to shorter time intervals before treatment have been reported. Although call-to-needle times can be very short when GPs or the ambulance staffs give thrombolytics pre-hospital, the interval from the onset of symptoms until the call for medical assistance varies widely from one patient to the other. Symptoms are often interpreted incorrectly. Event recorders and transtelephonic ECG recorders are increasingly used to improve decision making, but these systems are unable to capture transient ECG event such as infrequent arrhythmias or ischemic episodes. Moreover, all these systems require setting up new information technology infrastructures and medical services and need skilled personnel to interpret the ECG and take decisions for the patient care. This approach would be very impractical for patients with infrequent symptoms such as arrhythmias and ischemias that represent $85 \%$ of the cardiac diseased patients, and would be very expensive if adapted for every citizen at risk. The challenge is twofold:

- detect as early as possible the onset of ischemic events, even for citizens that have not yet any known cardiac disease.

- involve the health care structures without delay, but only if necessary.

The solution proposed by the European EPI-MEDICS project [7],[8] is to develop a novel, enhanced, portable and intelligent Personal ECG Monitor (PEM) for the early detection of cardiac ischemia and arrhythmia. It will be able to record pseudo-orthogonal 3-lead ECGs, store and derive standard 12-lead ECGs, incorporate intelligent self-adaptive serial ECG data processing and decisionmaking techniques, generate different levels of alarms, and forward the alarm messages with the recorded signals and the patient's Electronic Health Record (EHR) to the relevant health care providers by means of new generation wireless communication protocols (Bluetooth and GSM/GPRS).

\section{EPI-MEDICS use cases}

The device developed by the EPI-MEDICS consortium is a consumer device that can be bought by citizens in a drugstore. But it can also be recommended to a patient by his physician. Depending on the situation, the initialization will be different and the PEM will be used in either a generic way or a patient-specific way.

The PEM can generate three different levels of alarm. A major alarm corresponds to an acute infarction or a severe arrhythmia. In this case, the PEM shall directly send a message to the emergency call center. An informative (lower level) alarm consists in displaying an advice to the patient on the PEM screen such as the arrangement of an appointment with the attending physician. The medium alarm is a more complex scenario that is detailed in the following section.

\subsection{Medium alarm scenario}

A citizen/patient has just recorded an ECG with his PEM. The recorded PEM-specific ECG together with the health data entered by the citizen/patient are processed. In case of a borderline interpretation, the PEM will ask for additional information (symptoms). Decision-making takes also into account the patient's risk factors previously entered by the health professional and stored in the patient's health record, if any. In case a medium alarm situation has been detected (suspicion of ischemia and/or of bradycardia, ventricular tachycardia or other risky arrhythmias), the PEM connects to the patient's mobile phone via BlueTooth and sends an alarm message to a dedicated PEM server (Figure 1). Such a message contains the critical ECG (that is the last recorded ECG), a reference ECG (that corresponds to an ECG recorded in normal cardiac situation, if any), the alarm level (informative, medium, major), the coordinates of the user, his medical contacts, his relatives, the whole EHR stored in the PEM card. The message and the data are encoded in XML except for the ECGs that are encoded in the SCP-ECG format and encapsulated in the XML message.

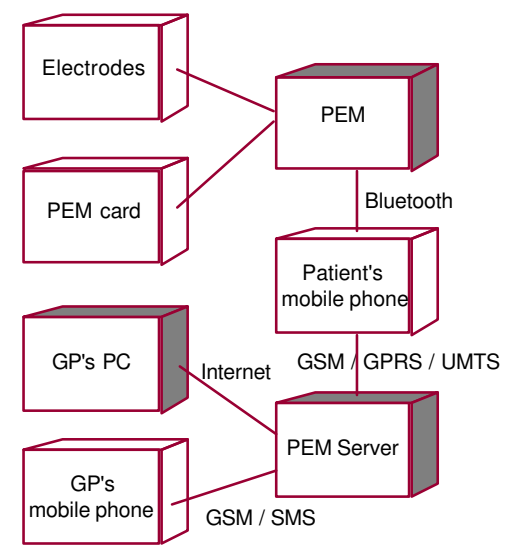

Figure 1. Deployment diagram for the medium alarm scenario. The PEM server is located in a secured central facility connected to Internet or to a secured health care Intranet such as the RSS in France.

The PEM server notifies this event to the patient's attending physician by a SMS (Short Message Service) and an email. A temporary web page presenting the alarm is also created. The GP receives the SMS message and calls the patient back to tell him that he handles the alarm. He then connects to Internet and reaches quickly the dedicated web page (secured http) which link is specified in the PEM server's email. He authenticates himself by entering the password that is part of the SMS message and reviews the alarm: recorded ECGs, user information, clinical history, clinical signs... $\mathrm{He}$ 
eventually calls the patient back to give him the result of his analysis or calls the emergency services if needed. Some time later, the PEM server erases the alarm web page.

\subsection{Routine visit scenario}

A routine visit could have been arranged after a PEM advice (informative alarm) for example. To get access to the information recorded and stored in the PEM device, the health professional firstly connects the PEM to his PC through an Internet browser and via the BlueTooth connection. All operations will be managed through the Web browser and the embedded web server. Once connected to the PEM device, the health professional can then:

- view and update the patient information: demographic data, clinical statements (ischemia...), additional information (blood pressure, cholesterol...),

- view, annotate, delete and download the ECGs recorded by the patient. He can also define the ECG that will be considered as reference for serial ECG analysis,

- adjust the decision making criteria according to the patient specificities (hypertrophy due to intensive sport...) or risk factors,

- upload the transformation matrix that derives standard 12-lead ECGs from the 3-lead PEM ECGs and which has been previously computed on the PEM application server with simultaneously-recorded PEM and standard ECGs,
- trigger a simulated medium alarm in order to reassure the patient and to show him how the PEM works in case of a medium alarm. A test of the technical infrastructure and parameters is also performed.

\section{PEM embedded web server}

One of the main barriers to the wide spread dissemination of health informatics solutions is the necessity to have specialized software to review the patient record. EPI-MEDICS clearly aims at developing pervasive solutions, such that any physician can review the data that have been collected and/or sent by the PEM device without requiring specialized software and/or the necessity to set-up specialized call centers. The solution we have designed is to embed a GoAhead web server in the PEM itself to facilitate the viewing and the update of the EHR, for example, during a routine visit at a health professional's office.

\subsection{Data model}

The PEM data layer is based on the data model shown in Figure 2. The ECG signals are stored as SCP-ECG [9] standard compliant files. The alarm messages and the EHR data are encoded in XML because it assures the web page independence in terms of presentation and language. The XML pages are transformed into HTML pages via XSLT. The PEM uses the XML parser and XSLT processor of the Apache group: Xerces and Xalan. The data are stored on the PEM SmartCard and retrieved via a proprietary file system.

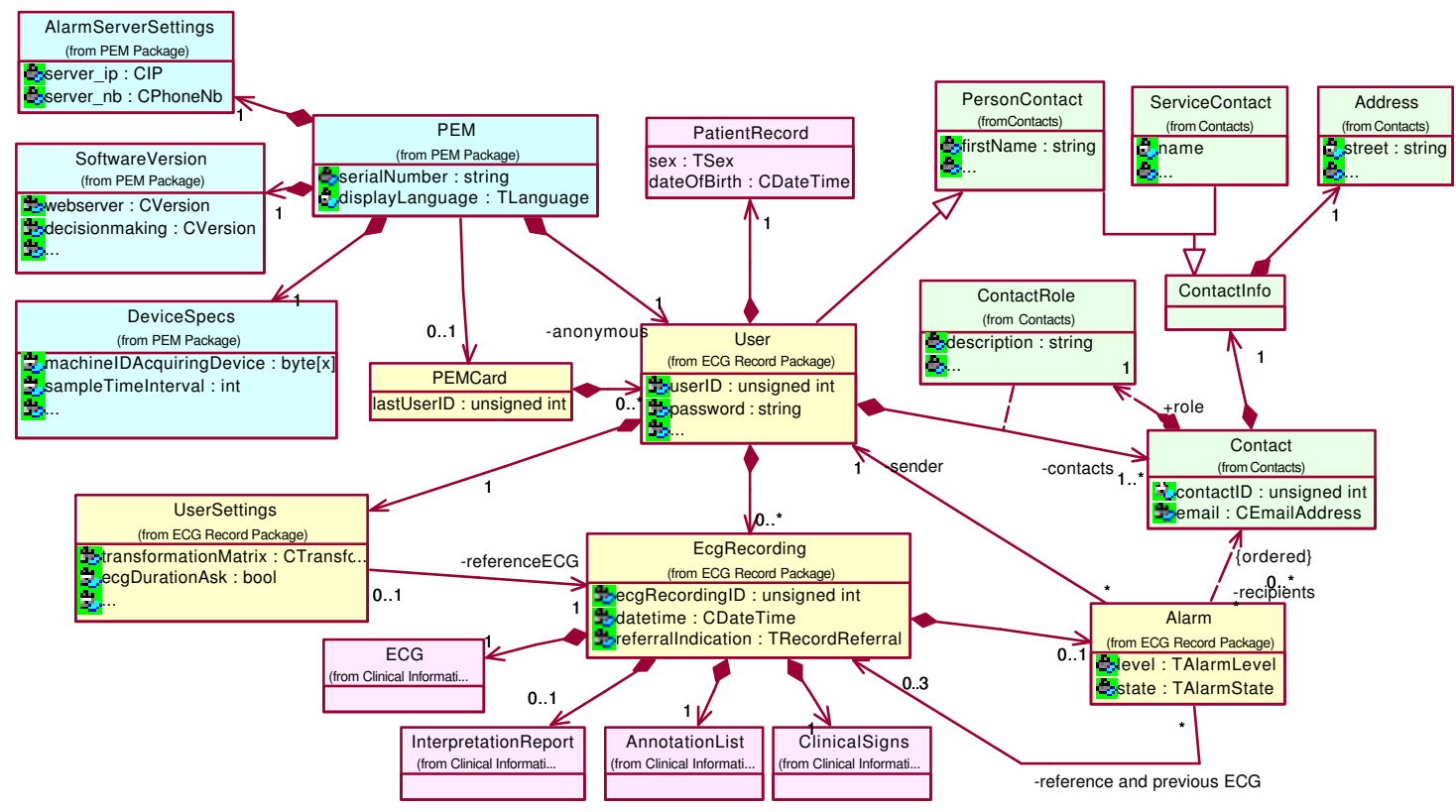

Figure 2. PEM data Model. A PEM card can support several users. Each user has demographic data and different types of contact (family, physicians). PEM ECGs are recorded, interpreted by decision-making programs and can generate alarms. 


\subsection{PEM web interface}

Once the PEM is connected to the PC, the user launches his Internet browser and specifies the PEM device address. The main page of the PEM website is displayed: the user can control the PEM through this usual and standard type of interface.

The user can be the citizen/patient owning the PEM device, a health professional or a technician. The information stored in the PEM are stratified according to a tree structure, with chapters and sub-chapters. Figure 3 presents the information relative to the patient, the ECG parameters and the clinical data.

Figure 4 presents the HTML web page displaying the parameters used for the calculation of the cardiovascular risk factor.

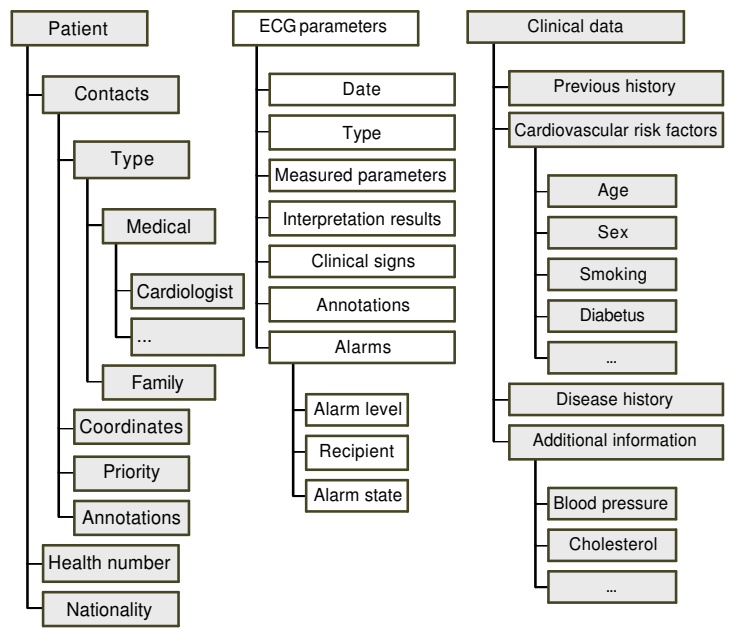

Figure 3. Data organized according to a tree structure.

\section{Conclusion}

The citizen/patient's needs are changing. They want to be more involved in the management of their own health, preferring painless methods of diagnostics and treatments. In the face of these changes, we need to develop pervasive systems that bring to all intelligence, speed, miniaturization and sophistication.

The European EPI-MEDICS consortium proposes such a pervasive solution that is based on an intelligent personal ECG monitor embedding professional capabilities in term of recording and processing. It is concretely cost saving: only a standard web browser and Internet connectivity are requested. No specific driver is needed on the PEM user's computer. The user manipulates the PEM device as he browses the web: he is in a familiar environment.

Furthermore, the care providers are involved only if necessary and no specific infrastructures are required: communications are based on standard external tools. Thus, healthcare will become personalized, wearable and ubiquitous.

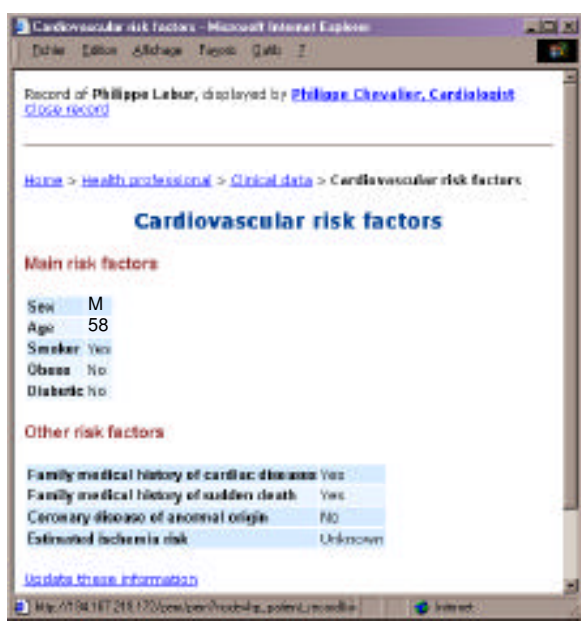

Figure 4. Web page presenting the cardiovascular risk factors stored in the PEM for the user named Lebur.

\section{Acknowledgements}

This work is supported in part by the EU, DGXIII, under Grant IST-2000-26164 EPI-MEDICS.

\section{References}

[1] Eysenbach G. Consumer health informatics. BMJ 2000;320:1713-1716.

[2] Hansmann U, Merk L, Nicklous MS, Stober T. 2001. Pervasive Computing Handbook. Springer-Verlag.

[3] European Community Information Society Technologies Advisory Group; Scenarios for Ambient Intelligence in 2010; Final Report, 2001, EC 2001: http://www.cordis.lu/ist/istag.htm

[4] Weiser M, Hot Topics: Ubiquitous Computing. IEEE Computer 1993;26:71-72.

[5] Smith R. The future of healthcare systems. BMJ 1997;314:1495-1496.

[6] Task Force report: the pre-hospital management of acute heart attacks. Recommendations of a Task Force of the European Society of Cardiology and The European Resuscitation Council. Eur. Heart J. 1998;19:1140-1164.

[7] Rubel P, Gouaux F, Fayn J, Assanelli D, Cuce' A, Edenbrandt L, Malossi C. Towards Intelligent and Mobile Systems for Early Detection and Interpretation of Cardiological Syndromes. In: Murray A, editors. Computers in Cardiology 2001. Piscataway, USA: IEEE Computer Society Press, 2001:193-6.

[8] EPI-MEDICS: a project of the European Commission (http://epi-medics.insa-lyon.fr).

[9] ENV 1064:1993 Medical Informatics - Standard Communication Protocol - Computer-assisted Electrocardiography (SCP-ECG), http://www.centc251.org

Address for correspondence.

F Gouaux.

INSERM XR121/ERM107

28, avenue du Doyen Lépine 69394 Lyon Cedex 3, France gouaux@insa.insa-lyon.fr 